\title{
A remark on the construction of designs for two-way elimination of heterogeneity
}

\section{Leon S. Sterling and Nicholas Wormald}

A method of construction of designs with parameters $v_{1}=r_{2}=p^{2}, r_{1}=v_{2}=p+1, b=p(p+1), k=p$ which may be used for the two-way elimination of heterogeneity is discussed. These designs were first studied in connection with estimating tobacco mosaic virus. Our designs have the advantage that every treatment occurs at most once in a row or column. We give the designs explicitly for $p=3,4,5$.

It is well known (see $\mathrm{Hall}[2, \mathrm{p} .176]$ ) that an affine plane or balanced incomplete block design with parameters $v=p^{2}, b=p(p+l)$, $r=p+1, k=p, \lambda=1$ exists whenever $p$ is a prime or a prime power. Moreover, the incidence matrix of this plane can always be chosen in the form

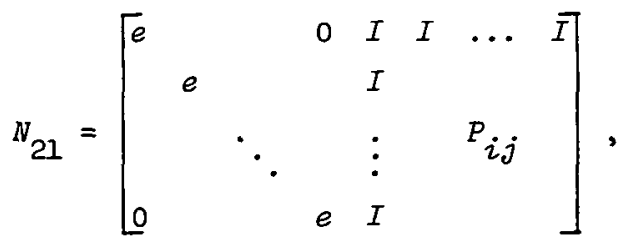

where $e$ is the $p \times I$ matrix of ones, $I$ is the identity matrix of order $p \times p$ and $P_{i j}$ are $p \times p$ permutation matrices. In fact if $p$

Received 10 February 1976. Communicated by Jennifer R.S. Wallis. The authors would like to thank Dr Jennifer Seberry Wallis for her help and encouragement. 
is prime we can choose $P_{i j}=T^{i j}$ where $T$ is a $p \times p$ matrix with 1 in the $(p, 1)$ element and for entries $(s, s+1)$ and zero elsewhere.

We choose $N_{31}=\left(J_{p+1}-I_{p+1}\right) \times e^{T}$, where $e^{T}$ denotes $e$ transposed, $J$ is the square matrix of ones, and $A \times B=\left(a_{i j} B\right)$ where $A=\left(a_{i j}\right)$.

Clearly $N_{31}$ is a balanced incomplete block design with parameters

$$
v=p+1, \quad b=p(p+1), \quad r=p^{2}, k=p, \lambda=p(p-1) .
$$

Further,

$$
\begin{aligned}
& N_{21} N_{21}^{T}=p I+J, \\
& N_{31} N_{31}^{T}=p I+p(p-1) J, \\
& N_{21} N_{31}^{T}=p J .
\end{aligned}
$$

So $N_{21}$ and $N_{31}$ have the required properties. The design construction is as follows :

CONSTRUCTION. Write the affine plane in the form of $h_{21}=\left[Q_{1} Q_{2} \ldots Q_{p+1}\right]$ where $Q_{i}, i=1, \ldots, p+1$ is a $p^{2} \times p(0,1)$ matrix with 1 non-zero element per row and $p$ non-zero elements per column. Now assign the $p+1$ treatments $v_{1}, v_{2}, \ldots, v_{p+1}$ to the nonzero elements of $N_{21}$ so that

(i) each treatment $v_{1}, v_{2}, \ldots, v_{i-1}, v_{i+1}, \ldots, v_{p+1}$ occurs exactly once in each column of $Q_{i}, i=1, \ldots, p+1$;

(ii) each treatment $v_{1}, v_{2}, \ldots, v_{p+1}$ occurs exactly once in each row of $N_{21}$.

The design is now obtained by labelling the rows of $N_{21}$ with treatments $V_{1}, V_{2}, \ldots, V_{p}{ }^{2}$, and writing $v_{j} v_{i}$ in the $k$ th column of the design if row $v_{j}$ of $N_{21}$ had $v_{i}$ assigned to the non-zero element 
in the $(j, k)$ position of $N_{21}$.

This method of choice ensures that each pair occurs once and only once in the final design. So $N_{23}=J$.

The $v_{1}, v_{2}, \ldots, v_{p+1}$ give the $\left(p+1, p(p+1), p^{2}, p, p(p-1)\right)$ brlanced incomplete block design while the $V_{1}, \ldots, V_{p^{2}}$ give the $\left(p^{2}, p(p+1), p+1, p, 1\right)$ balanced incomplete block design.

We note that the manner of choosing the latter design ensures that it is resolvable and that the blocks comprising one replication of its treatments all contain the same subset of $p$ treatments from the second set.

EXAMPLE. Let $a, b, c, d$ be the treatments from the $N_{31}$ matrix, and $A, B, C, D, E, F, G, H, I$ the treatments from the $N_{21}$ matrix. Consider

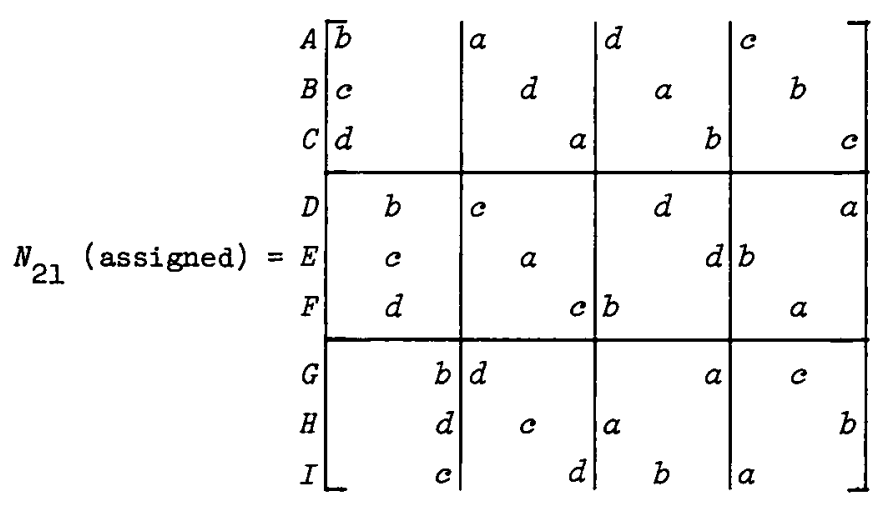

This has $b, c, d$ in each column of $Q_{1} ; a, c, d$ in each column of $Q_{2} ; a, b, d$ in each column of $Q_{3} ; a, b, c$ in each column of $Q_{4}$; and $a, b, c, d$ in each row of $N_{21}$.

Then the design with parameters

$$
v_{1}=r_{2}=9, r_{1}=v_{2}=4, b=12, k=3
$$

is 


$$
\begin{array}{llllllllllll}
A b & D b & G b & A a & B d & C a & A d & B a & C b & A c & B b & C c \\
B c & E c & H d & D c & E a & F c & F b & D d & E d & E b & F a & D a \\
C d & F d & I c & G d & H c & I d & H a & I b & G a & I a & G c & H b
\end{array} .
$$

Another solution with

$$
v_{1}=r_{2}=9, r_{1}=v_{2}=4, b=12, k=3
$$

is

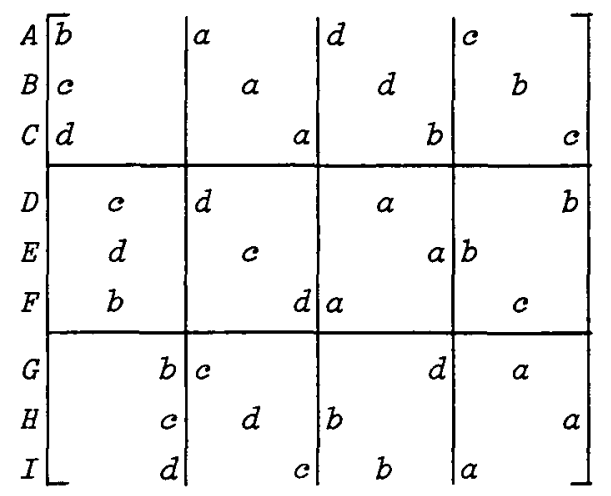

A solution for

$$
v_{1}=r_{2}=16, \quad r_{1}=v_{2}=5, b=20, k=4
$$

is

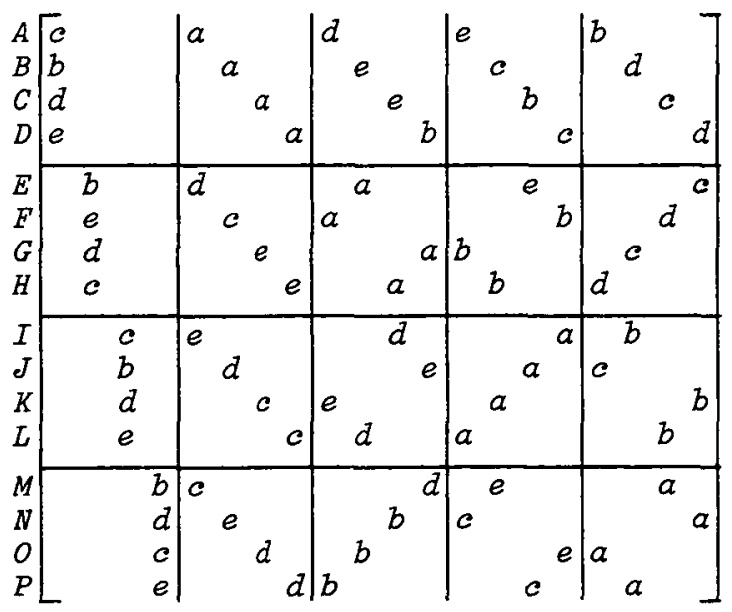

Table 1 gives one solution for

$$
v_{1}=r_{2}=25, r_{1}=v_{2}=6, b=30, k=5 .
$$




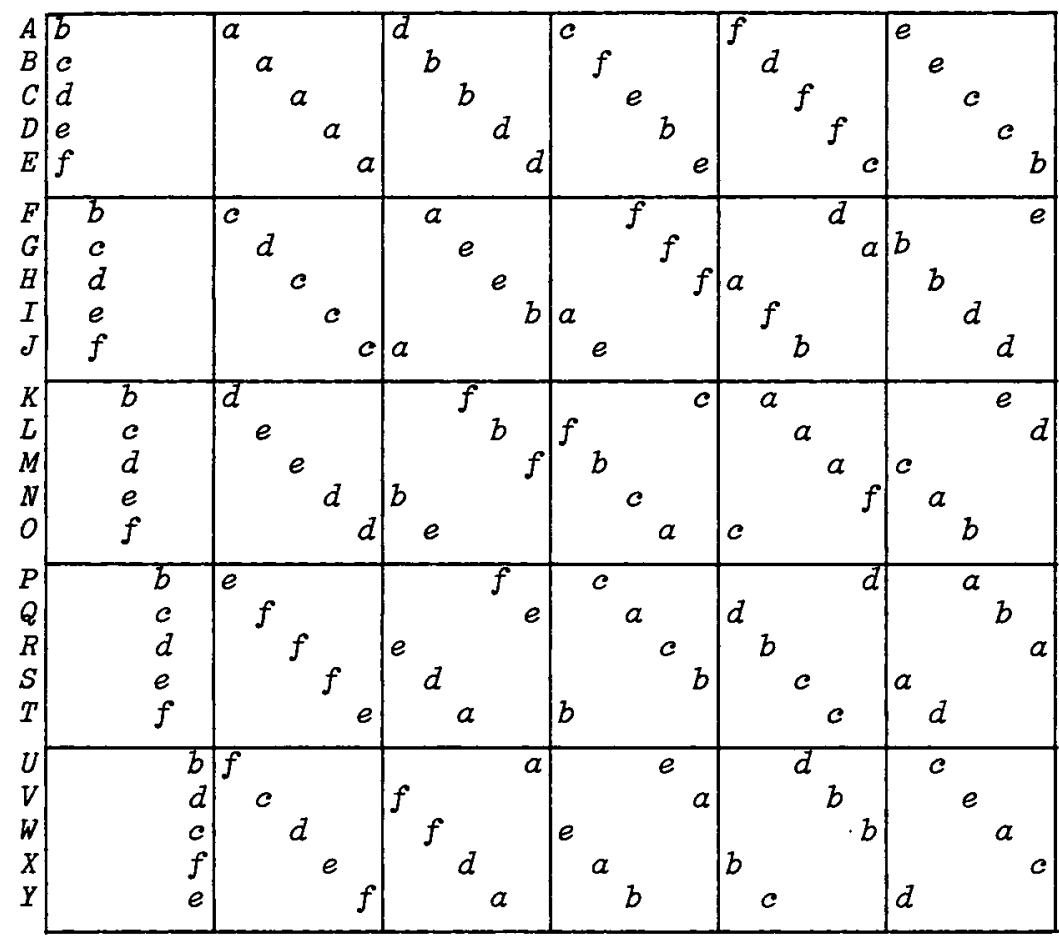

Table 1

We found many solutions for $k=3$, and for $k=5$, the results in the latter case being found by a computer search.

We also searched for solutions for $p=7$ using a computer but none were found in the time available.

\section{References}

[1] Hiralal Agrawal, "Some methods of construction of designs for two-way elimination of heterogeneity. I", J. Amer. Statist. Assoc. 61 (1966), 1153-1171.

[2] Marshall Hall, Jr., Combinatorial theory (Blaisdell [Ginn and Co.], Waltham, Massachusetts; Toronto, Ontario; London; 1967).

[3] D.A. Preece, "Non-orthogonal Graeco-Latin designs", Combinatorial mathematics IV (Proc. Fourth Austral. Conf., to appear). 
[4] W.J. Youden, "Use of incomplete block replications in estimating tobacco mosaic virus", Contributions from Boyce Thompson Institute 9 (1937), 41-48.

Department of Mathematics,

Institute of Advanced Studies,

Australian National University,

Canberra, ACT.

Present addresses:

Department of Mathematics,

University of Melbourne,

Parkville,

victoria;

Department of Mathematics,

University of Newcastle,

Newcastle,

New South Wales. 